\title{
SUGGESTION OF AN EQUATION OF MOTION TO CALCULATE THE DAMPING RATIO DURING EARTHQUAKE BASED ON A CYCLIC PROCEDURE
}

\author{
H. NADERPOUR \\ Semnan University, Faculty of Civil Engineering, Semnan, Iran \\ e-mail: naderpour@semnan.ac.ir \\ R.C. BARros \\ University of Porto(FEUP), Faculty of Engineering, Porto, Portugal \\ S.M. KHATAMI \\ Semnan University, Faculty of Civil Engineering, Semnan, Iran
}

\begin{abstract}
Large horizontal relative displacements are naturally caused by seismic excitation, which is able to provide collisions between two adjacent buildings due to insufficient separation distance and severe damages due to impacts, especially in tall buildings. In this paper, the impact is numerically simulated and two needed parameters are calculated, including the impact force and energy absorption. In order to calculate the mentioned parameters, mathematical study is carried out to model an unreal link element, which is logically assumed to be a spring and dashpot to determine the lateral displacement and damping ratio of the impact. For the determination of the dynamic response of the impact, a new equation of motion is theoretically suggested to the evaluate impact force and energy dissipation. In order to confirm the rendered equation, a series of parametric studies are performed and the accuracy of the formulas is confirmed.
\end{abstract}

Keywords: pounding, impact, dissipated rnergy, coefficient of restitution

\section{Introduction}

During earthquake, buildings commonly collide with each other due to different dynamic characteristics of adjacent buildings, insufficient gap between them and vibrate out of phase. This phenomenon is called "building pounding". The pounding is experimentally shown by an instance of rapid strong pulsation, which causes severe damage and is repeated by decreasing stiffness of the building after each collision. Consequently, as it is obviously seen, there are many tall buildings constructed with a small gap size. The effectiveness of pounding must be considered to avoid collisions or decline impact forces when adjacent buildings are designed and built. Importance of the mentioned subject has been understood by some researchers, who tried to report their studies about pounding. Anagnostopolos $(1995,1996,2004)$ was among the first researchers who explained possible dangers due to building pounding. He presented an equation of motion to calculate the impact damping ratio. Kasai and Maison (1997) presented a formulation and simulated multiple-degree-of-freedom equations of motion for floor-to-floor pounding between two 15-storey and 8-storey buildings. The influence of building separation, relative mass, and contact location properties were assessed by them. Jankowski (2008) carried out the most studies about pounding in two different terms, which were experimental and numerical analyses. Jankowski $(2009,2010,2012)$ suggested efficient methods to calculate the impact ratio by justifying different equations and also substantially implemented different experimental tests to predict impact velocity by focusing on dropping balls onto a rigid surface. Muthukumar and 
DesRoches (2006) introduced an equation of motion to generate an impact and determine the damping coefficient. Ye et al. (2009) and Yu and Gonzalez (2008) theoretically explained two equations of motions in order to determine the impact damping ratio by focusing on stiffness of the spring and also impact velocity. They coordinated a stereo-mechanical model with energy loss during the impact. Komodromos et al. (2007) expressed an equation of motion to create an impact between two bodies, which could estimate the impact damping ratio. The base isolation systems have recently been described by Komodromos and Polycarpou (2011, 2012) and the pounding was numerically investigated. Barros and Khatami (2012a) parametrically evaluated different damping equations to show the optimum formula to calculate the impact force. They also examined a new model of impact to coordinate the results among numerical and experimental studies (Barros and Khatami, 2013). Furthermore, the effectiveness of concrete shear wall was considered to reduce collision between adjacent buildings by Barros and Khatami (2012b). Naderpour et al. (2013) investigated results of all represented formulas and compared them in terms of dissipated energy. They also suggested an approximate trend to select the coefficient of restitution, which became equal with the impact velocity (Barros et al., 2013). Subsequently, a new equation of motion has been suggested to simulate the impact and figure damping terms out of collision (Naderpour et al., 2014). Nevertheless, it seems that there is lack of calculating the impact force and energy dissipation, which could be satisfied by selecting different coefficients of restitutions and various situations. It leads to introdution different equations with different results, and it can not be accepted. So, there is a need to use an equation which could be a response to all questions about the pounding. In this paper, a new mathematical program, called CRVK, submitted by Naderpour et al. (2014) at the FEUP, is specifically developed to model the impact between two bodies. Subsequently, an equation of motion is numerically suggested and the accuracy of the formula is confirmed by comparison between the dissipated energy and the energy loss.

\section{Past equations to calculate the impact damping ratio}

The contact element is an unreal element to model the impact between two bodies, which commonly includes a spring and dashpot and is called "Hertz contact element". It is widely used to calculate the impact force and energy absorption. The impact is parametrically modeled when the relative displacement exceeds the separation distance and the contact element is activated to simulate collision between two bodies and to calculate the impact force. For this challenge, an equation of motion is numerically considered to simulate the impact, which becomes

$$
F_{i m p}(t)=k_{s} \delta^{n}(t)+c_{d} \dot{\delta}(t)
$$

where $k_{s}$ is stiffness of the spring, $c_{d}$ denotes the damping ratio of the dashpot, $\delta$ and $\dot{\delta}$ describe the lateral displacement and velocity, respectively. The power of $n$ is recommended to be 1 or 1.5 , which depends significantly on the model.

In this equation, the damping ratio has been related by different equations which are individually investigated. In order to introduce the equations, the coefficient of restitution $(C R)$ is used, which is defined as the ratio of relative velocities before and after the impact, and is written as

$$
0<C R=\frac{\dot{\delta}_{\text {before }}}{\dot{\delta}_{\text {after }}}<1
$$


The first equation of motion was suggested by Anagnostopolos (2004) to calculate the damping ratio and solve equation (2.1) when the power of $n$ is 1

$$
c_{i m p}=2 \zeta \sqrt{k_{s} \frac{m_{i} m_{j}}{m_{i}+m_{j}}} \quad \zeta=-\frac{\ln (C R)}{\sqrt{\pi^{2}+[\ln (C R)]^{2}}}
$$

where $m_{i}, m_{j}$ are the colliding masses and $\zeta$ is the damping ratio. Here, the dashpot is still activated when two bodies are separated and the system shows a negative impact, which is not clearly justified.

Jankowski et al. (2009) introduced an equation to calculate the damping coefficient, which can be focused on the coefficient of restitution and solved it when $n=1.5$. He found that for the impact between two bodies, the negative force and dissipated energy have to disappear as it was pointed out. It was not inherently confirmed by physical explanation. Therefore, the impact force in the contact element may be expressed based on the given formula

$$
c_{i m p}=2 \zeta \sqrt{\beta \sqrt{\delta(t)} \frac{m_{i} m_{j}}{m_{i}+m_{j}}} \quad \zeta=\frac{9 \sqrt{5}}{2} \frac{1-C R^{2}}{C R[C R(9 \pi-16)+16]}
$$

where $\beta$ is the impact stiffness parameter depending on material properties of the colliding bodies.

Barros et al. (2013) simulated an impact and justified an equation to determine the impact damping ratio based on the coefficient of restitution. The equation yields better behavior in terms of the impact against equation $(2.3)_{2}$ and $(2.4)_{2}$, when the accuracy of the mentioned equation is compared with each other

$$
\zeta=\left(\frac{1-C R^{2}}{C R\left[\sqrt{\pi}\left(\frac{1}{2} C R+\frac{1}{\pi}\right)-C R\right]}\right)^{2}
$$

An improved equation of motion to find the damping ratio was considered by Muthukumar and DisRocher (2006). They believed that the damping ratio of the dashpot was not dependent on the contact element and the behavior of spring had an important effect on the coefficient of damping ratio. They presented a new equation, which depended on three parameters, including the coefficient of restitution, stiffness of the spring and impact velocity. They demonstrated that energy loss during the impact had to be equal by energy absorption during an impact. It is worth to mention that the power of $n$ was suggested to be 1.5

$$
c_{i m p}=\zeta \delta^{n} \quad \zeta=\frac{3 k_{s}\left(1-C R^{2}\right)}{4 \dot{\delta}_{i m p}}
$$

Ye et al. (2009) claimed that equation $(2.6)_{2}$ was incorrect to calculate the damping ratio for simulation of pounding in order to evaluate the impact between two buildings. Despite the mentioned point, three parameters used in equation $(2.6)_{2}$, the coefficient of restitution $(C R)$, stiffness of the spring and impact velocity were listed to present the impact damping ratio by Ye et al. (2009). They numerically indicated that energy loss during the impact should be equal to kinetic energy by the dashpot and is expressed as follows

$$
\zeta=\frac{8 k_{s}(1-C R)}{5 C R \dot{\delta}_{i m p}}
$$

In order to obtain equation (2.7), Ye et al. (2009) tried to use an approximate relation between lateral displacement and velocity to solve the relation and to confirm the suggested equation, an integration around a hysteresis loop was used. 
Naderpour et al. (2013) proposed another equation to show the impact between two bodies focusing on impact velocity, stiffness of the spring and the coefficient of restitution

$$
\zeta=\frac{2 k_{h}}{5 C R^{2} \dot{\delta}_{i m p}} \sqrt{\frac{1-C R}{1+C R}}
$$

Equation (2.8) completes equation (2.5) in calculating the impact force and energy dissipation.
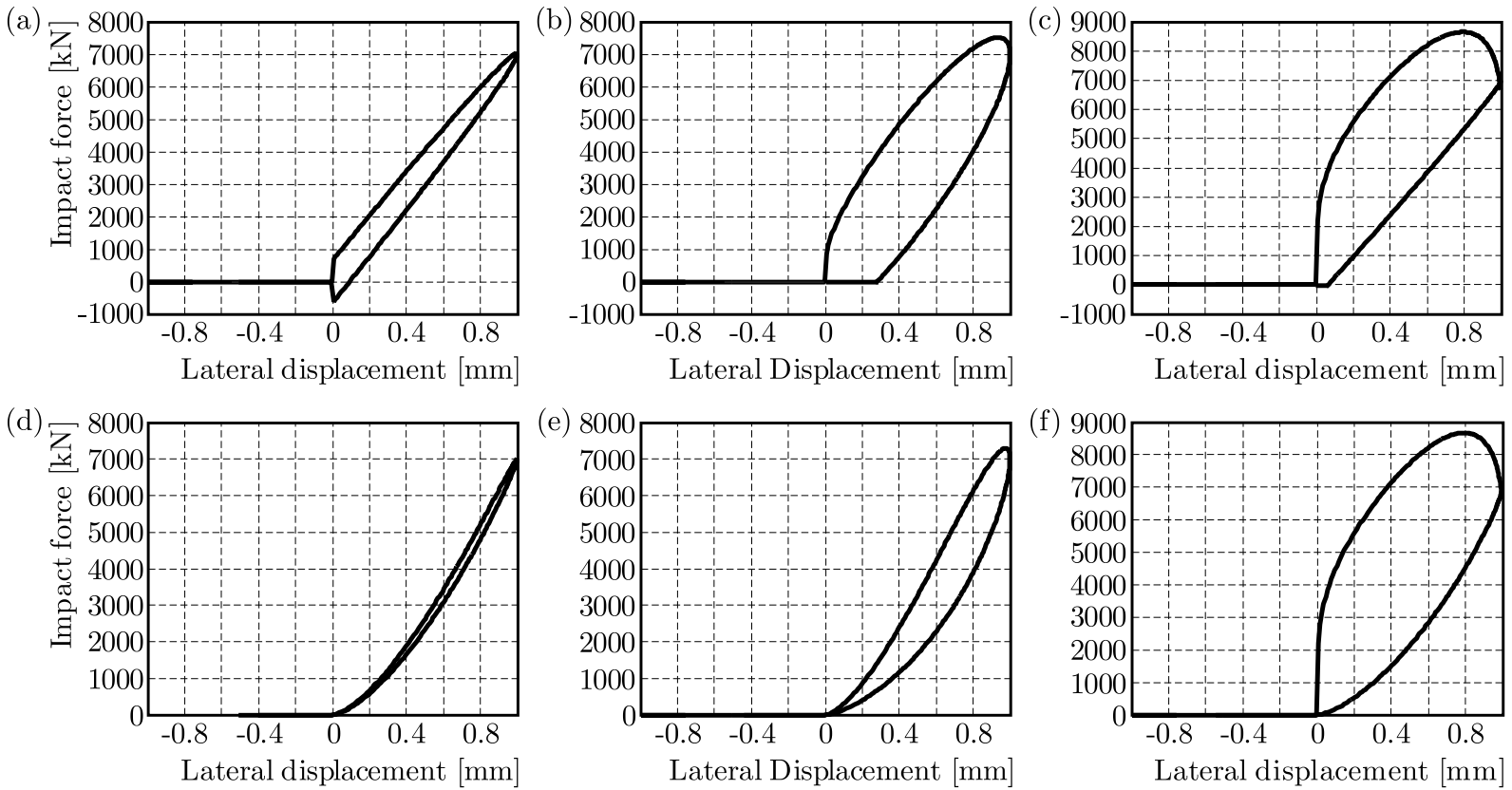

Fig. 1. Impact force versus lateral displacement based on: (a) Eq. $(2.3)_{2}$, (b) Eq. (2.4) $)_{2}$, (c) Eq. (2.5), (d) Eq. (2.6) $)_{2}$, (e) Eq. (2.7) and (f) Eq. (2.8)

\section{The proposed impact damping model}

As it has been noted, an efficient method by consideration a new equation in terms of the damping ratio is specially needed to illustrate an impact and solve the damping ratio based on all effectively used parameters in the impact such as lateral displacement, velocity, acceleration, stiffness of the spring, impact velocity and the coefficient of restitution. In order to define the impact damping ratio, which can be obtained from results of simulations and confirmed by numerical analyses, the impact between two bodies is equivalently modeled and a cyclic process for simulation of the structural pounding is numerically considered. In the current study, the impact is mathematically divided into three parts, during the impact, after the impact and when two bodies are completely separated from each other. In the first part, all elements are activated and energy can be absorbed by using the dashpot. In the second part, the dashpot is displayed and energy is not dissipated and, finally, any impact and energy are not shown as the bodies have been separated. For simulation of the impact and to remedy the equation of contact, using CRVK program (Naderpour et al., 2014), the spring and dashpot are activated when the impact takes place and, subsequently, the dashpot is automatically eliminated when the two bodies are detached. The three mentioned parts of the impact can be numerically expressed as below

$$
F_{i m p}= \begin{cases}k_{s} \delta^{n}(t)+c_{i m p} \dot{\delta}(t) & \text { for } \delta(t)>0 \text { and } \dot{\delta}(t)>0 \\ k_{s} \delta^{n}(t) & \text { for } \delta(t)>0 \text { and } \dot{\delta}(t)<0 \\ 0 & \text { for } \delta(t)<0\end{cases}
$$

where $n=1.5$. 
In order to determine the impact force and energy dissipation, the impact between two bodies is simulated and the hysteresis loop is depicted. It is assumed that the dissipated energy is approximately expressed by the enclosed area of the hysteresis curve due to the impact. On the other hand, the kinetic energy loss due to the impact was demonstrated by Goldsmith (1960), which was seen as

$$
E=\frac{1}{2} \frac{m_{i} m_{j}}{m_{i}+m_{j}}\left(1-C R^{2}\right) \dot{\delta}_{i m p}^{2}
$$

It is obviously confirmed that the dissipated energy during the impact has to be equal to the kinetic energy calculated by equation (3.2). Undoubtedly, if both energies becomes equal to each other, it shows the accuracy of the impact damping ratio.

As it has been described in the previous part of this paper, many researchers proposed different equations of motion to simulate collision between two bodies and calculate the impact force during earthquake records. As it was shown, all the equations describe the damping coefficient based on some parameters such as $C R$. It is obviously seen that each equation gives specific results by selecting $C R$ and the results are modified by using another $C R$. Consequently, it cannot be accepted and the results can not be also confirmed.

In order to provide a new equation in terms of the damping ratio, an unknown parameter is considered to be $c_{i m p}$, which depends on some parameters as

$$
c_{i m p} \cong\left\{k_{s}, m, C R, \dot{\delta}_{i m p}, \delta, \dot{\delta}, \ddot{\delta}\right\}
$$

To meet this challenge, an unreal link element is considered to be at the level of bodies, which includes a spring and a dashpot to calculate lateral displacement and energy absorption, respectively.

The damping coefficient is defined by the following expression

$$
c_{i m p}=\zeta_{i m p} k_{s} \delta^{n}(t)
$$

where $k_{s}$ is stiffness of the spring and $\delta(t)$ denotes lateral displacement $(n=1.5)$. The impact damping ratio is given by different terms, and becomes

$$
\zeta_{i m p}=w_{C R} \frac{1-C R}{\ddot{\delta}(t) \dot{\delta}(t) \delta(t)} C R_{i m p}
$$

In order to solve equation (3.5), the terms need to be presented based on the mentioned parameters, seen in $c_{i m p}$. So it is estimated to be

$$
C R_{i m p(i)}=\rho(i) C R^{\eta}
$$

In equation (3.5), $w_{C R}$ depends on the impact velocity, which is determined as below

$$
w_{C R(i)}=\alpha(i) \dot{\delta}_{i m p}^{\beta}
$$

In order to start the simulation of the impact and solve the program to get the impact damping ratio, a value of mass is given and CRVK program calculates lateral displacement, velocity and acceleration of the spring streched between two bodies. Stiffness of the spring is determined and a $C R$ is also selected. The program solves the equations and calculates energy dissipation, which is equal to the area of the hysteresis loop of each impact and compares it with the kinetic energy calculated by equation (3.2). Both energies should be equal if all factors has been correctly selected.

This process is numerically repeated for all considered coefficients of restitution for solving the equations, and the results are frequently compared to confirm equation (3.5). 
Firstly, let us know the value of equal masses that is calculated by equation (3.8). Having used the mass, stiffness of the spring is estimated by the given figure

$$
m=\frac{m_{i} m_{j}}{m_{i}+m_{j}}
$$

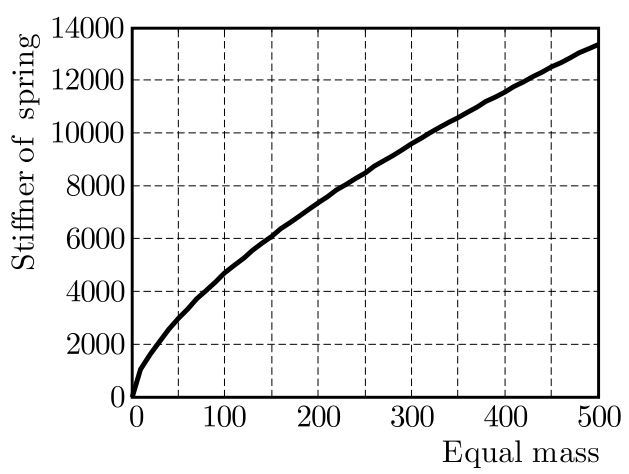

Fig. 2. Stiffness of the spring based on equal masses

Now, the coefficient of restitution $(C R)$ is randomly selected $(0<C R<1)$. In particular, for $C R=0$ and 1, we have full energy for a perfectly plastic impact and no energy to show an elastic impact, respectively. After selecting $C R$ and having impact velocity, the coefficient of $w_{C R}$ is calculated based on the selected $C R$.
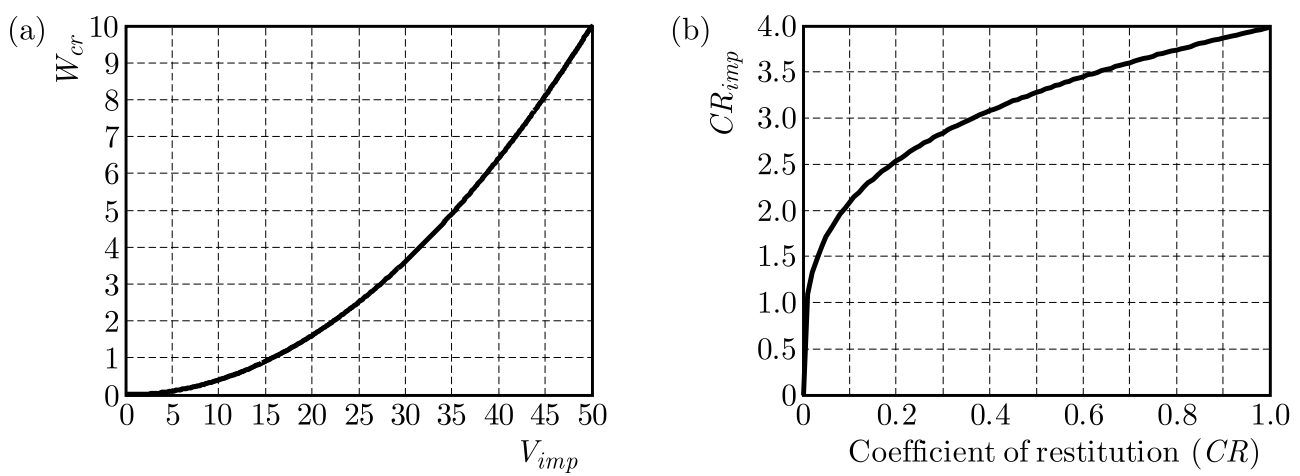

Fig. 3. (a) Coefficient $w_{C R}$ based on impact velocity, (b) coefficient $C R_{i m p}$ based on the coefficient of restitution

Now, after selecting $C R$ and getting $w_{C R}$, by making use of Fig. 3, $C R_{i m p}$ is determined.

Based on the energy equilibrium before and after an impact and the energy loss mentioned in equation (3.2) and using all parameters and all $C R \mathrm{~s}$, a cyclic process is provided to calculate the kinetic energy loss and available energy. By comparing these energies with each other, one gets an approximate damping term. In order to show a better image from the mentioned cyclic process of calculation of the impact damping ratio, a chart is rendered to determine the impact damping ratio based on all used parameters.

The chart is divided into two different parts. In the first step, it is assumed that the damping coefficient depends on the acceleration, velocity, lateral displacement and also the coefficient of restitution. For this challenge, a value of $C R$ is selected and $C R_{i m p}$ is estimated. The hysteresis loop is depicted and also energy absorption $A_{h}$ is calculated. Kinetic energy and energy absorption are compared with each other, if both become equal, then $C R_{i m p}$ has been correctly selected and the system selects a new $C R$ and $C R_{i m p}$ automatically.

Finally, the impact damping ratio is also described as

$$
\zeta_{i m p}=0.0159 \dot{\delta}_{i m p}^{2} \frac{1-C R}{\ddot{\delta}(t) \dot{\delta}(t) \delta(t)} C R^{0.2805}
$$



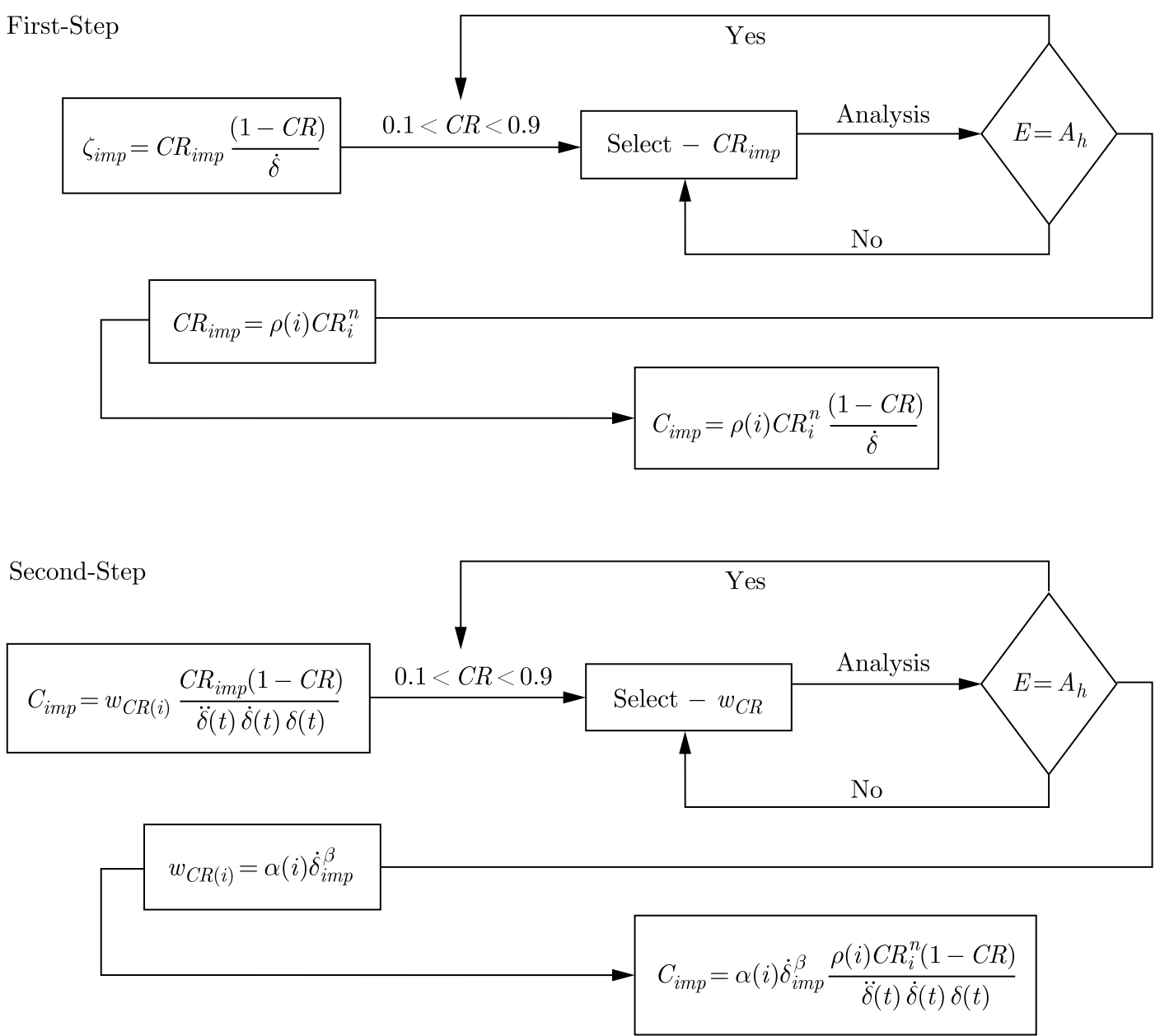

Fig. 4. The iterative procedure to determine the damping coefficient

For example, two SDOF systems are considered which are separated by a $0.2 \mathrm{~mm}$ gap from each other. The assumed lateral displacement by the impact velocity equal to $10 \mathrm{~m} / \mathrm{s}$ is defined, and equal mass of $100 \mathrm{~kg}$ and $C R=0.4$ are taken into account, respectively. So we have: $m=100 \mathrm{~kg}$, $k=6000 \mathrm{kN} / \sqrt{\mathrm{mm}}, \dot{\delta}_{i m p}=10 \mathrm{~m} / \mathrm{s}, C R=0.4, C R_{i m p}=1.1903, w_{C R}=2.5269$.

(a)

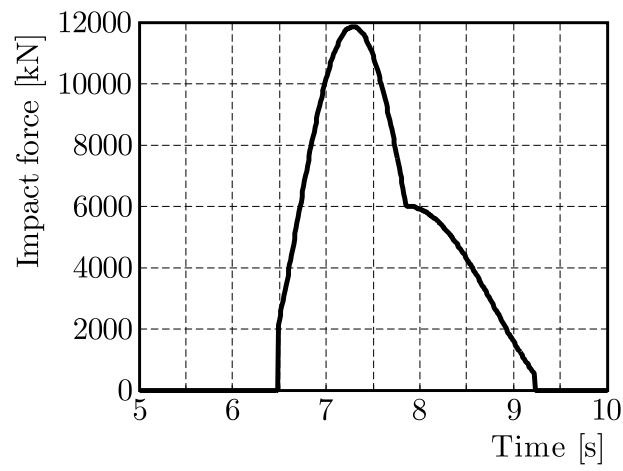

(b)

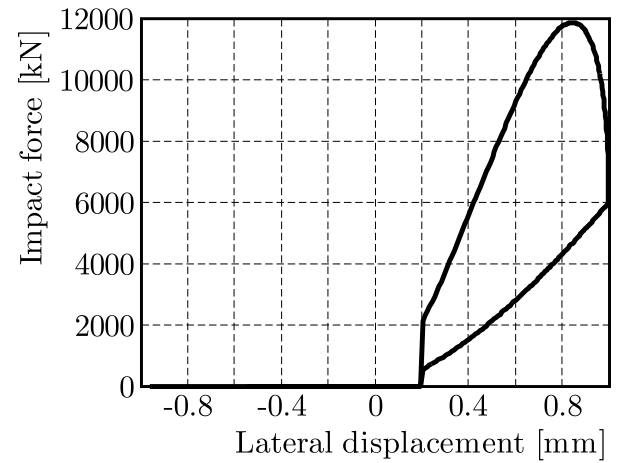

Fig. 5. Impact force versus time and lateral displacement

As it has been shown, the maximum impact force is approximately $12000 \mathrm{kN}$, and the dissipated energy, which has already been assumed to be the area of the hysteresis loop is $4189 \mathrm{kN} \cdot \mathrm{mm}$. On the other hand, the kinetic energy is calculated to be $4200 \mathrm{kN} \cdot \mathrm{mm}$ based on the Goldsmith rule (Eq. (3.5)). It is obviously achieved that both energies are close to each other and the 
calculated $C R$ is 0.3527 , which shows an error about $0.007 \%$, which is negligible. Based on this process, all $C R \mathrm{~s}$ are selected and compared as depicted in Fig. 6.

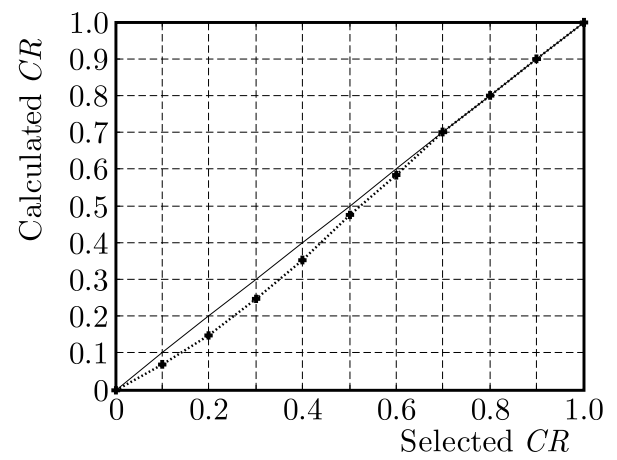

Fig. 6. Accuracy of the proposed damping ratio by numerical analysis

In order to investigate the accuracy of equation (3.9), an experimental test has been carried out to compare the results of experiment and numerical analysis.

Katija et al. (2006) carried out an impact test between two steel frames, each frame of $300 \mathrm{~kg}$ with a $40 \cdot 40 \mathrm{~cm}$ beam separated by a $10 \mathrm{~cm}$ gap. The collision test was implemented using a horizontal hydraulic high-speed loading machine. This machine had a loading capacity of $1000 \mathrm{kN}$ and a maximum loading speed of $3.0 \mathrm{~m} / \mathrm{s}$. The collision test was carried out in a line on a guide rail with length of $3000 \mathrm{~mm}$. Two static and hysteresis tests were calibrated by using CRVK program when the impact velocity was $0.68 \mathrm{~m} / \mathrm{s}$ for the second evaluation.

Both tests are considered and numerically examined to investigate the impact between two the bodies. Equation (3.9) is used and the proposed impact model is also defined to calibrate and compare the results of experimental and numerical analyses.

(a)

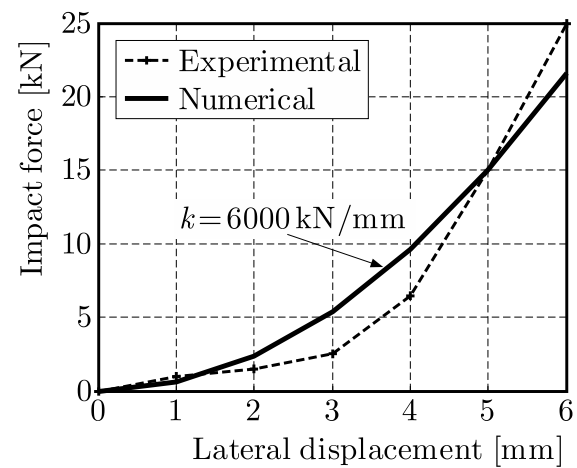

(b)

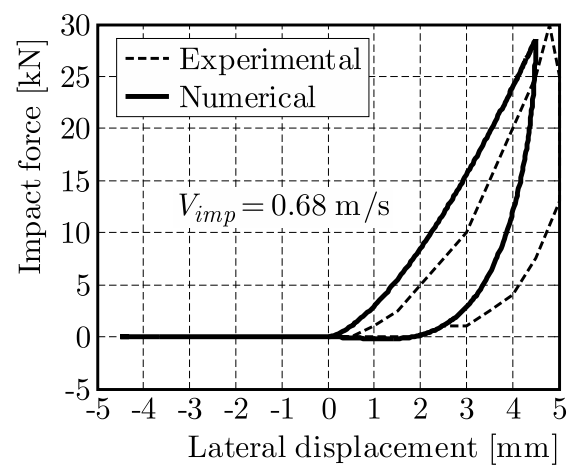

Fig. 7. Accuracy of the proposed damping ratio by calibrating the numerical and experimental analyses

The accuracy of the mentioned equation is confirmed as the trend of both tests is similar, and the maximum impact forces are 29.85 and $28.32 \mathrm{kN}$, which shows an error about $5 \%$, which is negligible.

\section{Numerical study}

A parametric study is considered in order to describe the proposed impact damping model. The impact between two bodies is numerically simulated to measure the impact force and dissipated energy during seismic excitation. For this challenge, CRVK program is basically used and developed to perform dynamic analyses under Parkfield (1966), San Fernando (1971), Kobe (1995) and El Centro (1940) earthquake records. These records have different content of excitation 
frequencies, different random magnitude of accelerations in time and different earthquake durations. Besides, their place of occurrence and geological conditions are distinct. All mentioned records are directly normalized to investigate the effect of earthquake properties when bodies collide with each other.

\section{Effect of gap size}

In order to investigate the effect of separation distance, the gap size is varied from 0 to $20 \mathrm{~cm}$. The link element between the bodies is automatically activated when the gap size exceeds from the considered separation distance between them. Figure 8 depicts the effect of separation distance on the response in terms of the impact force during the four earthquake records. In particular, the curves follow an irregular decrease in the gap size 0 to $20 \mathrm{~cm}$ and the impact forces are suddenly reduced in San Fernando and Parkfield records and are slightly declining in the two other records. Therefore, an increase in the separation distance shows an effective decline in the impact force due to collision between the two bodies.

(a)

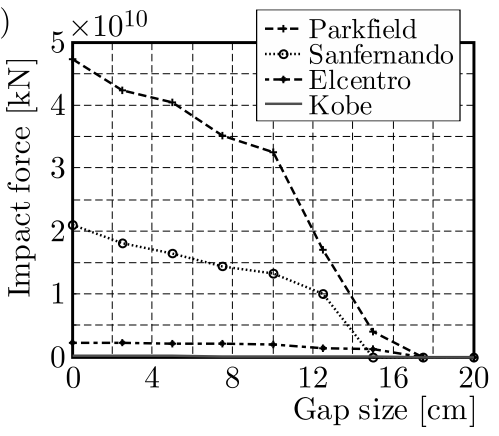

(b)

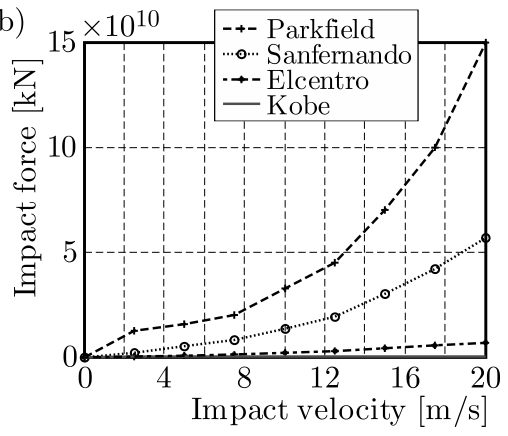

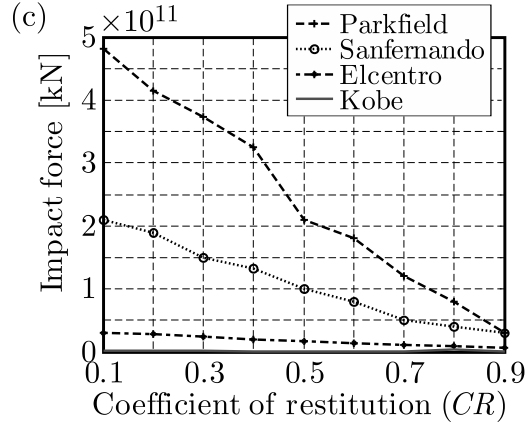

Fig. 8. Impact forces with the increasing (a) gap size, (b) impact velocity and (c) coefficient of restitution

\section{Effect of coefficient of restitution}

As the coefficient of restitution has a great effect on calculation of the impact damping ratio, different values of $C R$ are considered to compare the impact forces during earthquakes. Figure 8 shows that the peak impact forces slowly decrease when the coefficient of restitution increases. A similar trend of impact force responses based on the coefficient of restitution is observed as the effect of $C R$ is numerically seen to be linear in the proposed impact damping model. For instance, San Fernando record shows an impact force about $21 \cdot 10^{9} \mathrm{kN}$ and $6.7 \cdot 10^{9} \mathrm{kN}$ for $C R=0.1$ and 0.9 , respectively.

\section{Effect of impact velocity}

As it is shown in equations (3.5) and (3.6), the impact damping ratio directly depends on the impact velocity. Figure 8 shows a calm increase with growth of the impact velocity, which seems to be predictable. In order to get the responses of impacts and compare the results of maximum impact forces, different values of the impact velocity are considered from the interval 0 to $20 \mathrm{~m} / \mathrm{s}$, and the impact is simulated by different velocities. For example, the impact forces are $7.2 \cdot 10^{10}, 3.13 \cdot 10^{10}, 0.41 \cdot 10^{1} 0$ and $0.019 \cdot 10^{10} \mathrm{kN}$ for $15 \mathrm{~m} / \mathrm{s}$ of the impact velocity in the Parkfield, San Fernando, El Centro and Kobe, respectively. 


\section{Conclusion}

When two buildings are built close to each other, it is very important to consider pounding phenomena between them due to earthquake. Researchers have introduced an unreal element to calculate the impact force and the dissipated energy during seismic excitation by making use of the spring and the dashpot. Different equations of motion are presented to determine the damping ratio and an estimated value of the impact between the two colliding bodies. Here, a new equation based on all effectiveness parameters has been suggested, and the accuracy of the formula has been numerically evaluated. The effect of stiffness of the spring, impact velocity, coefficient of restitution and also separation distance have been investigated in detail. A parametric analysis has been carried out to show the results of the impact force and dissipated energy which have been then compared with the kinetic energy to confirm the created formula.

\section{Refrences}

1. Anagnostopoulos S.A., 1995, Earthquake induced pounding: state of the art, Proceedings of 10th European Conference on Earthquake Engineering, Balkema, Rotterdam, 897-905

2. Anagnostopoulos S.A., 1996, Building pounding re-examined: how serious a problem is it? Eleventh World Conference on Earthquake Engineering, Pergamon, Elsevier Science Ltd.

3. Anagnostopoulos S.A., 2004, Equivalent viscous damping for modeling inelastic impacts in earthquake pounding problems, Earthquake Engineering and Structural Dynamics, 33, 8, 897-902

4. Barros R.C, Khatami S.M., 2012a, Building pounding forces for different link element models, CST2011, 4-7 September, Dubrovnic, Croatia

5. Barros R.C, Khatami S.M., 2012b, Seismic response effect of shear walls in reducing pounding risk of reinforced concrete building pounding subjected to near fault-ground motion, 15th World Conference Earthquake Engineering, 15WCEE, Lisbon, Portugal

6. Barros R.C., Khatami S.M., 2013, Damping ratios for pounding of adjacent building and their consequence on the evaluation of impact forces by numerical and experimental models, Mecânica Experimental, 22, 119-131

7. Barros R.C., Naderpour H., Khatami S.M., Mortezaei A.R., 2013, Influence of seismic pounding on rc buildings with and without base isolation system subject to near-fault ground motions, Journal of Rehabilitation in Civil Engineering, 1, 39-52

8. Goldsmith W., 1960, Impact: The Theory and Physical Behavior of Colliding Solids, 1st ed., Edward Arnold: London U.K.

9. JANKowski R., 2008, Earthquake-induced pounding between equal height buildings with substantially different dynamic properties, Engineering Structures, 30, 10, 2818-2829

10. JANKowski R., 2009, Non-linear FEM analysis of earthquake-induced pounding between the main building and the stairway tower of the Olive View Hospital, Engineering Structures, 31, 8, 1851-1864

11. Jankowski R., 2010, Experimental study on earthquake-induced pounding between structural elements made of different building materials, Earthquake Engineering and Structural Dynamics, 39, 3, 343-354

12. JANKowski R., 2012, Non-linear FEM analysis of pounding-involved response of buildings under non-uniform earthquake excitation, Engineering Structures, 37, 0, 99-105

13. Kajita Y., Kitahara T., Nishimoto, N., 2006, Estimation of maximum impact force on natural rubber during collision, First European Conference on Earthquake Engineering and Seismologhy, 1st Ecees, Geneva, Switzland 
14. Kasai K., Maison B.F., 1997, Building pounding damage during the 1989 Loma Prieta earthquake, Engineering Structures, 19, 3, 195-207

15. Komodromos P., Polycarpou P., 2011, Numerical investigation of potential mitigation measures for poundings of seismically isolated buildings, Earthquake and Structures, 2, 1, 1-24

16. Komodromos P., Polycarpou P., 2012, A nonlinear impact model for simulating the use of rubber shock absorbers for mitigating the effect of structural pounding during earthquake, Earthquake Engineering and Structural Dynamics 42, 81-100

17. Komodromos P., Polycarpou P.C., Papaloizou L., Phocas M.C., 2007, Response of seismically isolated buildings considering poundings, Earthquake Engineering and Structural Dynamics, 36, 12, 1605-1622

18. Muthukumar S., DesRoches R., 2006, A Hertz contact model with non-linear damping for pounding simulation, Earthquake Engineering and Structural Dynamics, 35, 7, 811-828

19. Naderpour H., Barros R.C, Khatami S.M., 2013, A new equation of motion to calculate the impact force and the energy dissipation, Proceedings of the Fourteenth International Conference on Civil, Structural and Environmental Engineering Computing, B.H.V. Topping, P. Iványi (Edit.), Civil-Comp Press, Stirlingshire, UK, Paper 92, Doi:10.4203/ccp.102.92

20. Naderpour H., Barros R.C., Khatami S.M., 2014, A new model for calculating the impact force and the energy dissipation based on cr-factor and impact velocity, Scientia Iranica, 1, 48-63

21. Ye K., Li L., ZHu H., 2009, A note on the Hertz contact model with nonlinear damping for pounding simulation, Earthquake Engineering and Structural Dynamics, 38, 9, 1135-1142

22. Yu Q.K., Gonzalez D., 2008, Lessons learned from the October 15, 2006 Hawaii earthquake and the August 15, 2007 Peru earthquake, 14th World Conference on Earthquake Engineering: Innovation Practice Safety, International Association for Earthquake Engineering 